\title{
EPIGENETICS
}

\section{WIP1 creates hush and havoc}

The protein phosphatase $2 \mathrm{C}$ family member WIP1, a serine and threonine phosphatase encoded by $P P M 1 D$, is overexpressed in several cancer types, but how it contributes to disease formation is unclear. Dmitry Bulavin and colleagues have found that WIP1 is involved in regulating DNA methylation-mediated silencing of heterochromatin and that it might contribute to C-to-T substitutions and mutation load in breast cancers.

Bulavin and colleagues found that Ppm1d knockout male mice have reduced fertility, and as Ppm1d is highly expressed in the testis, the authors examined spermatogenesis in these mice. The Ppm1d-null germ cells had increased areas of condensed heterochromatin that included silenced repetitive DNA elements, such as L1 LINES (long interspersed nuclear elements). Knockdown of WIP1 using small interfering RNAs (siRNAs) in two cancer cell lines, HCT116 and MCF7, also reduced expression from repetitive DNA elements. These elements had increased levels

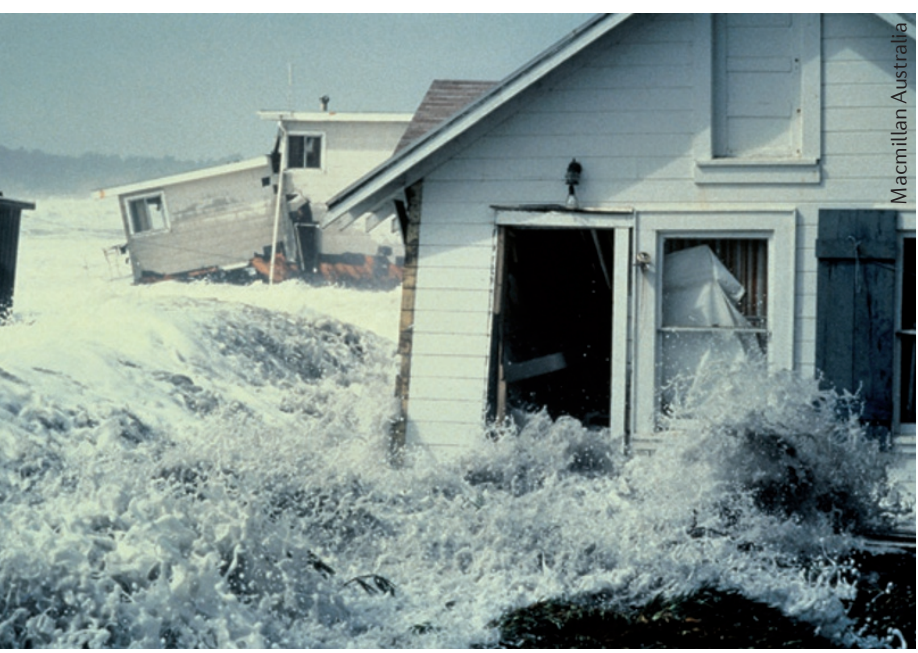

of histone modifications associated with repressive chromatin and a decrease in the histone modifications associated with active chromatin. Moreover, DNA methylation levels were also altered: an antibody that binds to 5-methyl cytosine (5meC) showed that levels of $5 \mathrm{meC}$ were increased at L1 LINEs in WIP1-knockdown cells.

What causes this increase in DNA methylation in the absence of WIP1? WIP1 regulates several DNA repair pathways, and mice lacking WIP1 show constitutive activation of the ataxia telangiectasia mutated (ATM) and ataxia telangiectasia and RAD3-related protein (ATR) DNA repair pathways in the absence of DNA damage. The authors examined the ATM kinase and found that ATM phosphorylation levels were increased in the absence of WIP1, as were the phosphorylation levels of 53BP1 and BRCA1, which are two downstream targets of ATM. BRCA1-targeting siRNAs increased expression of mRNAs from L1 LINEs and this was associated with reduced levels of $5 \mathrm{meC}$ at these DNA sites. Examination of DNA cytosine-5-methyltransferase $3 \mathrm{~A}$ (DNMT3A), DNMT3B and DNMT1 showed that DNMT3B and DNMT1 could bind to L1 LINE sequences in a BRCA1 dependent manner. Further chromatin immunoprecipitation assays showed that ATM-dependent phosphorylation of BRCA1 resulted in the formation of a complex that included heterochromatin protein $1 \gamma$ (HP1 $\gamma$; also known as CBX3) and DNMT3B, and that HP1 $\gamma$ enrichment at L1 LINE sites was dependent on BRCA1. Loss of ATM signalling inhibited DNA methylation of repetitive DNA sequences, indicating that loss of WIP1 and activation of an ATM-BRCA1 pathway results in expanded tracts of heterochromatin.

Re-expression of WIP1 in WIP1-knockdown cell lines led to a re-expression of repetitive DNA sequences, a decrease in $5 \mathrm{meC}$ levels and an increase in the levels of histone 3 lysine 4 trimethylation (H3K4me3). H3K4me3 is recognized by activation-induced cytidine deaminase (AID), which is an enzyme known to be involved in the generation of DNA point mutations. Having shown that AID is recruited to DNA in a WIP1-dependent manner, the authors speculated that increased expression of WIP1 could lead to the generation of C-to- $\mathrm{T}$ mutations because AID activity can result in the conversion of cytosine to uracil. Although this is normally repaired by the base excision repair (BER) pathway, WIP1 is an inhibitor of this pathway. Thus, if uracil is not replaced with cytosine through the BER pathway, at cell division a uracil will be paired with an adenine and the uracil will eventually be replaced by a thymine. The authors examined microarray and somatic mutation data sets from breast cancers, which often have PPM1D amplifications. The occurrence of C-to-T mutations correlated with PPM1D copy number. Analysis of mRNA expression levels of PPM1D and members of the APOBEC and AID deaminase family showed a strong correlation between C-to-T mutation load and expression levels of $P P M 1 D, A P O B E C 3 A$ and APOBEC $3 B$.

Thus, WIP1 could be an important mediator of point mutations in breast cancer in which it is overexpressed, and inhibition of this phosphatase could have important effects on the silencing of heterochromatin.

Nicola McCarthy

ORIGINAL RESEARCH PAPER Filipponi, D. et al. WIP1 controls global heterochromatin silencing via ATM/BRCA1-dependent DNA methylation. Cancer Cell 24, 528-541 (2013) 